\title{
Magneto-Hydrodynamic Antenna Design and Development Analysis with prototype
}

\author{
Rajveer S Yaduvanshi \\ Electronic and Communication \\ Deparment, \\ AIT, Govt of Delhi \\ India-110031 \\ E mail: yaduvanshirs@yahoo.co.in
}

\author{
Harish Parthasarathy \\ Electronic and Communication \\ Deparment, \\ NSIT, Govt of Delhi \\ India-110075 \\ E mail-harishp@nsit.ac in
}

\author{
Asok De \\ Principal \\ AIT, Govt of Delhi \\ India-110031 \\ E mail: asok.de@gamail.com
}

\begin{abstract}
A new class of antenna based on magnetohydrodynamic technique is presented. Magnetohydrodynamic Antenna, using electrically conducting fluid, such as $\mathrm{NaCl}$ solution under controlled electromagnetic fields is formulated and developed. Fluid resonator volume and electric field with magnetic field decides the resonant frequency and return loss respectively to make the antenna tuneable in the frequency range 4.5 to $9 \mathrm{GHz}$. The Maxwell's equations, Navier Stokes equations and equations of mass conservation for the conducting fluid and field have been set up. These are expressed as partial differential equations for the stream function electric and magnetic fields, these equations are first order in time. By discretizing these equations, we are able to numerically evaluate velocity field of the fluid in the near field region and electromagnetic field in the far field region. We propose to design, develop, formulate and fabricate an prototype MHD antenna [1-3]. Formulations of a rotating fluid frame, evolution of pointing vector, permeability and permittivity of MHD antenna have been worked out. Proposed work presents tuning mechanism of resonant frequency and dielectric constant for frequency agility and configurability. Measured results of prototype antenna possess return loss up to $-51.1 \mathrm{~dB}$ at $8.59 \mathrm{GHz}$ resonant frequency. And simulated resonant frequency comes out to be10.5GHz.
\end{abstract}

Keywords- Frequency agility, reconfigurability, MHD, radiation pattern, saline water.

\section{INTRODUCTION}

MHD antenna uses fluid as dielectric. The word magneto hydrodynamics (MHD) is derived from magneto- meaning magnetic field, and hydro- meaning liquid, and -dynamics meaning movement. MHD is the study of flow of electrically conducting liquids in electric and magnetic fields [3-5]. Here we have developed and tested magneto-hydrodynamic prototype antenna with detailed physics. Ting and King determined in 1970 that dielectric tube can resonate. To our knowledge no work has been done on MHD antenna as described here. Based on our own developed theory, we have proposed this prototype model with return loss results. Fluid antenna has advantage of shape reconfigurability and better coupling of electromagnetic signal with the probe, as no air presents in between [12]. We have developed physics as per equations (1-12) for electromagnetic wave coupling with conducting fluid in presence of electric and magnetic field. Design and testing stages of MHD antenna is shown as per figs. 1-13. Here, we demonstrate, how the directivity, radiation resistance and total energy radiated by this magnetohydrodynamic antenna can be computed, by the elementary surface integrals. We have developed, equations for rotating frame of conducting fluid, velocity field, electric field, magnetic field, pointing vector, current density, permittivity, permeability and vector potentials to realise an MHD Antenna [6-8].We have used saline water, ionised with DC voltage applied with the help of electrodes, in presence permanent magnetic field. Fluid acts as radiating element in the PPR (propylene random copolymer) cylindrical tube. SMA connector is used to supply RF input. Volume and shape of the fluid decides the resonant frequency. Excellent results of radiation parameters were reported on measurements of return loss and radiation pattern by the prototype, as listed in tables 1-5. We have divided this paper into five parts. First part consists introduction of MHD antenna system. Second part deals with formulations [9-11]. Section three focuses on brief explanation of the prototype development. Fourth section speaks about working of prototype system. Section five describes conclusion possible applications and scope of future work.

\section{FORMULATIONS}

\section{A. Motion of fluid in rotating frame}

The equation of motion of a fluid in a uniformly rotating frame with angular velocity $\omega$ is given by

$v, \mathrm{t}+v \cdot \nabla_{v}+2 \omega \mathrm{x} v+\omega \mathrm{x}(\omega \mathrm{x} \mathrm{r})=-\frac{\nabla_{p}}{\rho}+$ $v \nabla^{2} \vec{v}$

Assuming the flow to be two dimensional and fluid to be incompressible, obtain an equation for the stream function.

Velocity of fluid is given below

$v=v_{x}(\mathrm{t}, \mathrm{x}, \mathrm{y}) \hat{x}+v_{x}(\mathrm{t}, \mathrm{x}, \mathrm{y}) \hat{y}$

Angular velocity

$\omega=\omega_{0} \hat{z}$

The equation 
$\mathrm{v}_{\mathrm{x}, \mathrm{x}}+\mathrm{v}_{\mathrm{y}, \mathrm{y}}=0$

It gives

$$
v_{x}=\psi, y(\mathrm{t}, \mathrm{x}, \mathrm{y})
$$

$=-\psi, x(\mathrm{t}, \mathrm{x}, \mathrm{y})$

for some scalar function $\psi$ called the stream function.

As we know vortisity

$\Omega=\nabla \times v$

$$
=-\nabla^{2} \psi \hat{z}
$$

Using this in the equation obtained by taking the curl of the Navier Stokes equation, we have

\section{$\nabla^{2} \Omega$}

$\Omega, \mathrm{t}+\nabla(\Omega \times v)+2 \nabla \times(\omega \times v)+\nabla \times(\omega(\omega \times \mathrm{r}))=v$

Note that

$\omega \times(\omega \times \mathrm{r})=(\omega \cdot \mathrm{r}) \omega-\omega^{2} r$

so that

$\nabla \mathrm{x}(\omega \mathrm{x}(\omega \mathrm{x} \mathrm{r}))=0$

Since $\omega$ is assumed to be constant, thus, the Navier Stokes

equation gives

$$
\begin{aligned}
& \Omega, \mathrm{t}+\nabla \mathrm{x}(\Omega \times v)+2 \nabla \times(\omega \times v) \\
& =v \nabla^{2} \Omega
\end{aligned}
$$

\section{B. Far field radiation Pattern}

Space here r-radius, $\theta$ - Angle of elevation, $\phi$ - azimuth angle, $\omega_{1}, \omega_{2}$ are first and second components of the frequency and $\mathrm{r}, \phi, \theta$ are spherical co-ordinates.

$$
\begin{gathered}
0 \leq \theta \leq 180(\pi \mathrm{rad}) \\
0 \leq \phi \leq 360(2 \pi \mathrm{rad}) \\
\mathrm{r}=\sqrt{x^{2}+y^{2}+z^{2}} \\
\theta=\operatorname{Cos}^{-1} \frac{z}{r} \\
\phi=\operatorname{Tan}^{-1} \frac{y}{x} \\
\mathrm{x}=\mathrm{r} \operatorname{Sin} \theta \operatorname{Cos} \phi \\
\mathrm{y}=\mathrm{r} \operatorname{Sin} \theta \operatorname{Sin} \phi \\
\mathrm{z}=\mathrm{r} \operatorname{Cos} \theta
\end{gathered}
$$

$\mathrm{v} x \mathrm{~B}$ shall provide pointing vector in case of fluid. $\mathrm{E} \times \mathrm{H}$ gives pointing vector, here $\mathrm{H}$ vector to embed $\mathrm{v}$ effect due to conducting fluid . $E_{\theta}, H_{\phi}, E_{\phi}, H_{\theta}$ are electric and magnetic fields of MHD antenna and the pointing vector shall have the effect of conducting fluid velocity generated due to E, Radiation pattern shall depend on average radiated power. Any spherical coordinate triplet $(\mathrm{r}, \theta, \varphi)$, specify single point of three space coordinates in radiation field.

$$
\begin{gathered}
E_{\theta}=-\frac{\delta A_{0}}{\delta t}-\frac{\delta \phi}{d \theta} \\
E_{\phi}=-\frac{\delta A_{\phi}}{\delta t}-\frac{1}{\mathrm{r} \operatorname{Sin} \theta} \frac{\delta \phi}{d \theta} \\
E_{\theta}=-\frac{\delta A_{\theta}}{d t} \\
E_{\phi}=-\frac{\delta A_{\phi}}{d t}
\end{gathered}
$$

$$
\text { also } H_{\theta}=\frac{1}{\mu} \quad \vec{\nabla} \times \vec{A}=\left|\begin{array}{ccc}
\frac{\hat{r}}{\mathrm{r}^{2} \operatorname{Sin} \theta} & \frac{\hat{\theta}}{\mathrm{r} \operatorname{Sin} \theta} & \frac{\hat{\phi}}{\mathrm{r}} \\
\frac{\delta}{\delta r} & \frac{\delta}{\delta \theta} & \frac{\delta}{\delta \phi} \\
A_{r} & \mathrm{r} A_{\theta} & \mathrm{rSin} \theta A_{\phi}
\end{array}\right|
$$

Solution of above matrix shall provide us

$$
\frac{\widehat{\theta}}{\mathrm{r} \operatorname{Sin} \theta}\left(\frac{\delta A_{r}}{\delta \phi}-\frac{\delta}{\delta \mathrm{r}} \operatorname{Sin} \theta A_{\phi}\right)+\frac{\hat{\phi}}{\mathrm{r}}\left(\frac{\delta r A_{\theta}}{\delta \mathrm{r}}-\frac{\delta A_{\mathrm{r}}}{d \theta}\right)
$$

Hence

$$
\begin{gathered}
H_{\theta}=-\frac{1}{\mathrm{r} \operatorname{Sin} \theta} \frac{\delta}{\delta \mathrm{r}}\left(\mathrm{r} \operatorname{Sin} \theta A_{\phi}\right)=-\frac{\delta A_{\phi}}{\delta r} \\
H_{\phi}=\frac{1}{\mathrm{r}} \frac{d}{d \mathrm{r}}\left(\text { r. } A_{\theta}\right)=\frac{\delta A_{\theta}}{d r}
\end{gathered}
$$

$$
\left(\begin{array}{lll}
E & X H
\end{array}\right)_{\sigma}=E_{\theta} H_{\phi}-E_{\phi} H_{\theta},
$$

\section{(Resulting Pointing Vector)}

On substitution

Pointing vector $=-\frac{\delta A_{\theta}}{\delta t} \frac{\delta A_{\theta}}{\delta r}-\frac{\delta A_{\phi}}{d t} \frac{\delta A_{\phi}}{d r}$

Where $A_{\theta}=\frac{\psi(\mathrm{r}, \phi, \mathrm{t})}{\mathrm{r}}-\frac{\psi, r}{\mathrm{r}}$.

\section{And}

$\int_{0}^{\pi} \int_{0}^{2 \pi}(E x H) . \sigma r^{2} \operatorname{Sin} \theta \mathrm{d} \theta \mathrm{d} \phi$, shall provide pointing vector of radiator.

And

$\mathrm{J}=\sigma(\mathrm{E}+\mathrm{V} \times \mathrm{B})$ shall be the resultant of MHD antenna system, we need to calculate $\mathrm{E}$ at a given frequency. Here, first and second component of vector potentials are.

$$
\begin{aligned}
A_{1} & =\frac{\mu}{4 \pi} \sigma \frac{\int_{V} \vec{E}\left(\overrightarrow{r^{\prime}}, \omega\right) e^{-\frac{j \omega\left|\vec{r}-\vec{r}^{\prime}\right|}{c}}}{\left|\vec{r}-\vec{r}^{\prime}\right|} \mathrm{d}^{3} r^{\prime} \\
\text { or } A_{1} & =\frac{\mu}{4 \pi r} \sigma \int_{V} \vec{E}\left(\overrightarrow{r^{\prime}}, \omega\right) e^{-\frac{j \omega}{c} \vec{r} \cdot r^{\prime}} \mathrm{d}^{3} r^{\prime}
\end{aligned}
$$

And second component

$$
\begin{aligned}
& A_{2}= \\
& \frac{\mu}{4 \pi} \sigma \frac{\int_{V} \vec{v}(\vec{r}, \omega) X \vec{B}\left(\vec{r}, \omega-\omega_{1}\right) e^{-\frac{j \omega\left|\vec{r}-\vec{r}^{\prime}\right|}{c}}}{\left|\vec{r}-\vec{r}^{\prime}\right|} \mathrm{d}^{3} r^{\prime} \mathrm{d} \omega_{1}
\end{aligned}
$$

or

$$
A_{2}=\frac{\mu}{4 \pi r} \sigma e^{-\frac{j \omega}{c} r} \int_{V} \vec{v}\left(\overrightarrow{r^{\prime}}, \omega_{1}\right)
$$

$X \vec{B}\left(\overrightarrow{r^{\prime}}, \omega-\omega_{1}\right) d \omega_{1} e^{\frac{j \omega}{c} \widehat{r} \cdot r^{\prime}} \quad \mathrm{d}^{3} r^{\prime}$, with the help of $A_{1}$ and $A_{2}$, we can evaluate total magnitude of radiated energy 
per unit frequency per unit volume. This spectral density can be evaluated by applying Parseval's theorem (mathematics of DFT). As electric field $\mathrm{E}=-\mathrm{j} \omega A_{1 \theta} \widehat{\theta}-\mathrm{j} \omega A_{1 \phi} \widehat{\phi}$

$$
\begin{aligned}
E_{\theta} & =-\mathrm{j} \omega\left(A_{1 \theta}+A_{2 \theta}\right) \\
H_{\phi} & =-\frac{\mathrm{j} \omega}{v}\left(A_{1 \phi}+A_{2 \phi}\right),
\end{aligned}
$$

Here $\mathrm{H}_{\phi}$ embeds the velocity component of fluid at a given frequency. Now we shall evaluate $A_{1 \theta}$ and $A_{2 \theta}$ to compute energy spectral density. On integration we can evaluate total radiated energy. Also, we shall work to find $\mathrm{x}$, $\mathrm{y}, \mathrm{z}$ component of pointing vector.

Where r' denotes source and $r$ denotes far field distance. $E_{\theta}$ at a large distance shall contribute for $\eta=\mu / \mathcal{E}$ for plane wave propagation.

$A_{1 \theta}$ and $A_{2 \theta}$ should be function of $(\theta, \phi, \omega)$

also $\hat{\theta}=\hat{\phi} \mathrm{X} \hat{r}$ from spherical coordinates

$E_{\theta} \mathrm{X} H_{\phi}$ shall provide us the pointing vector of the radiated field

Here $\frac{1}{r}$ component reside in $\phi$, we need to calculate $\theta$ component to enable us far field component $E_{\theta}$ at large distance, also

$\eta=\frac{\mu}{\epsilon}$ for plane wave. We can thus evaluate total energy radiated.

We have $\hat{r}=\hat{\theta}$, and after normalization $\operatorname{Sin} \theta$ termgets cancelled.

(E x B) pointing vector for $\mathrm{x}, \mathrm{y}, \mathrm{z}$ components and taking $e^{-j \omega t}$ as common, we can evaluate $E_{x}, E_{y}, E_{z}$.

$$
=-j \omega A_{1 \theta} \hat{\theta}-j \omega A_{1 \Phi} \widehat{\Phi}
$$

Our objective is to evaluate total energy radiated per unit frequency per unit volume.

$$
\begin{aligned}
& E_{\theta}=-j \omega\left(A_{1 \theta}+A_{2 \theta}\right) \\
& \mathrm{H} \phi=\frac{-\mathrm{j} \omega}{\eta}\left(A_{1 \phi}+A_{2 \phi}\right), \text { here effect fluid velocity v have }
\end{aligned}
$$
been embedded in $\mathrm{H}$ field

$$
\frac{1}{2} \operatorname{Re}\left[E_{\theta} \mathrm{H} \phi\right]=\int \frac{\omega^{2}}{\eta} \operatorname{Re}\left(A_{\theta} A_{\phi}\right) d \omega r^{2} \operatorname{Sin} \theta \mathrm{d} \theta \mathrm{d} \phi
$$

assuming real part will effectively contribute.

$\hat{r}=\hat{x} \operatorname{Cos} \phi \operatorname{Sin} \theta+\hat{y} \operatorname{Sin} \phi \operatorname{Sin} \theta+\hat{z} \operatorname{Cos} \theta$

$\hat{\theta}=\frac{\delta \hat{r}}{\delta \theta}=\hat{x} \operatorname{Cos} \phi \operatorname{Cos} \theta+\hat{y} \operatorname{Sin} \phi \operatorname{Cos} \theta-\hat{z} \operatorname{Sin} \theta$

$$
\widehat{\phi}=\frac{\delta \hat{r}}{\delta \phi}=-\hat{x} \operatorname{Sin} \phi \operatorname{Sin} \theta+\hat{y} \operatorname{Cos} \phi \operatorname{Cos} \theta
$$

Hence

$$
\begin{gathered}
A_{1 \theta}=\frac{e^{-j \frac{\omega}{C} r}}{r}\left[\frac { \mu \sigma } { 4 \pi } \int \left(E_{x}\left(\overrightarrow{r^{\prime}}, \omega\right) \operatorname{Cos} \phi \operatorname{Cos} \theta+\right.\right. \\
E_{y}\left(\overrightarrow{r^{\prime}}, \omega\right) \operatorname{Sin} \theta \operatorname{Sin} \phi+E_{z}\left(\overrightarrow{r^{\prime}}, \omega\right) \operatorname{Cos} \theta
\end{gathered}
$$

$$
e^{-j \frac{\omega}{C}\left(x^{\prime} \operatorname{Cos} \theta \operatorname{Cos} \phi+y^{\prime} \operatorname{Sin} \theta \operatorname{Sin} \phi+z^{\prime} \operatorname{Cos} \theta\right.} d x^{\prime} d y^{\prime} d z^{\prime}
$$

$$
\text { And } \begin{aligned}
A_{2 \theta}= & \frac{e^{-j \frac{\omega}{C} r}}{r}\left\{\frac{\mu \sigma}{4 \pi} \int v_{\phi}\left(\overrightarrow{r^{\prime}}, \omega\right)\right. \\
& B_{r}\left(\overrightarrow{r^{\prime}}, \omega-\omega_{1}\right)-v_{r}\left(\overrightarrow{r^{\prime}}, \omega_{1}\right) \\
& \left.B_{\phi}\left(\overrightarrow{r^{\prime}}, \omega-\omega_{1}\right) e^{-j \frac{\omega}{C} \hat{r} \vec{r}} d^{3} r^{\prime}\right\}
\end{aligned}
$$

Hence, pointing vector can be defined as

$$
\begin{gathered}
E_{\theta}=-\mathrm{j} \omega \frac{e^{-j \frac{\omega}{C} r}}{r}\left(A_{1 \theta}(\theta, \phi, \omega)+A_{2 \theta}(\theta, \phi,\right. \\
\omega) \\
\text { And } \\
H_{\phi}=-\mathrm{j} \omega \frac{e^{-j \frac{\omega}{C} r}}{r}\left(A_{1 \phi}(\theta, \phi, \omega)+\right. \\
\left.A_{2 \phi}(\theta, \phi, \omega)\right)
\end{gathered}
$$

Hence energy spectral density

$$
\begin{aligned}
& \mathrm{D}=-\mathrm{j} \omega \int \omega^{2} \operatorname{Re}\left(A_{1 \theta}+A_{2 \theta}\right) \\
& \left(\overrightarrow{A_{1 \phi}}+\overrightarrow{A_{2 \phi}}\right) \operatorname{Sin} \theta \mathrm{d} \theta \mathrm{d} \phi
\end{aligned}
$$

Energy Spectral Density be evaluated by applying Parseval's Theorem

$$
\begin{gathered}
\int f(t) g(t) d t=\frac{1}{2 \pi} \int_{\text {or }}^{\cdot} \hat{f}(\omega), \hat{g}(\omega) d \omega \\
\int \vec{E}(\vec{r}, t) \times \vec{H}(\vec{r}, t) \hat{x} d s d t \quad(10)
\end{gathered}
$$

This shall provide us total energy radiated by the MHD antenna system.

$$
D=\frac{1}{2 \pi} \int \cdot \vec{E}(\vec{r}, \omega) \times \vec{H}(\vec{r}, \omega) \hat{x} d s d \omega
$$

\section{Permeability of MHD antenna}

We evaluate permeability of MHD antenna taking, conductivity and permittivity as constant. Hence $\mu$ becomes function of polynomial

In (E ,H, v) in MHD system, where E electric filed, h magnetic field and $v$ velocity of the fluid. Here $p, q, r$ are integers.

And $\mathrm{a}=1,2,3$

$$
\sum_{p, q, r, 0}^{N} \mu\left[\begin{array}{lllllllll}
p_{1} & p_{2} & p_{3} & q_{1} & q_{2} & q_{3} & r_{1} & r_{2} & r_{3}
\end{array}\right]
$$

$$
E_{1}{ }^{p_{1}} \quad E_{2}^{p_{2}} E_{3}^{p_{3}} H_{1}^{q_{1}} \quad H_{2}^{q_{2}} \quad H_{3}^{q_{3}} v_{1}^{r_{1}} \quad v_{2}^{r_{2}} v_{3}^{r_{3}}
$$

From Maxwell's equation, we have 
$\vec{\nabla} \times \vec{E}=-\frac{\delta(\mu \mathrm{H})}{\delta t}=-(\mu, \mathrm{t} \widehat{H}+\mu \widehat{H}, t)$

And, $\mu, \mathrm{t}=\sum_{a=1,2,3}\left(\mu_{, \mathrm{E}_{\mathrm{a}}} E_{a, t}+\mu_{, \mathrm{H}_{\mathrm{a}}} \mathrm{H}_{\mathrm{a}, \mathrm{t}}+\right.$ $\left.\mu_{, \mathrm{v}_{\mathrm{a}}} v_{a, t}\right)^{\prime}$

Thus, we observe that, permeability $\mu, \mathrm{t}$ becomes coupled function of $\mathrm{E}, \mathrm{H}$ and $\mathrm{v}$.

We can minimize the difference or error $\left(\mathrm{H}-H_{d}\right)$ with variational method, here $\mathrm{p}, \mathrm{q}, \mathrm{r}$ are integers, $H_{d}$, Desired outcome and $a=1,2,3$. We derive the relationship of $\mu$, permeability as function of $\mathrm{E}, \mathrm{H}$ and $\mathrm{v}$, where $\mathrm{E}$, electric field applied , $\mathrm{H}$ magnetic field and $\mathrm{v}$ is velocity field .Also $A_{1}, A_{2}$ are weight or magnitude of the function and $\overrightarrow{\lambda_{1}} \overrightarrow{\lambda_{2}}$ Lagrange multipliers. For3D analysis we have, $H_{\phi}(\mathrm{t}, \mathrm{x}, \mathrm{y}, \mathrm{z})$, $E_{\phi}(\mathrm{t}, \mathrm{x}, \mathrm{y}, \mathrm{z}), \mathrm{H}(\mathrm{t}, \mathrm{r}), \mathrm{E}(\mathrm{t}, \mathrm{r})$

$$
\begin{gathered}
A_{1} \int_{0}^{T} \int_{V}\left|\vec{H}-H_{d}\right|^{2} d t d^{3} r+A_{2} \int_{0}^{T} \int_{V}\left|\vec{E}-E_{d}\right|^{2} d t d^{3} r \\
+\int_{[0, T] x V}\left(\vec{\nabla} X \vec{E}-\frac{\delta}{\mathrm{dt}}(\mu \vec{H})\right.
\end{gathered}
$$

Hence on taking inner product and Lagrange multiplier in variational method, we get,

$S\left[\vec{H}, \vec{E}, \overrightarrow{\lambda_{1}}, \overrightarrow{\lambda_{2}}\right], \mu$ permeability is hidden in $\mathrm{H}$ value.

As an example, Let $\mathrm{F}\left(x_{1} \ldots \ldots \ldots x_{n}\right)$ and

$g_{i}\left(x_{1} \ldots \ldots \ldots x_{n}\right)=0$

Where

$\mathrm{i}=1,2, . . \mathrm{r}$,

$$
\begin{aligned}
F\left(x_{1}, \ldots x_{n}\right)+\sum_{i=1}^{r} & \lambda_{i} g_{i}\left(x_{1} \ldots \ldots x_{n}\right) \\
& =S\left(x_{1} \ldots \ldots x_{n}, \lambda_{1} \ldots \ldots \lambda_{r}\right)
\end{aligned}
$$

This gives the error value by variational method $\lambda_{1}, \lambda_{2}$ Lagrangian multiplier

$A_{1}, A_{2}$ are magnitude or function weight

$\mathrm{H}, E$ satisfy Maxwell's equation .

This way, we can minimize the difference or error $\left(\mathrm{H}-H_{d}\right)$, and $\mathrm{E}, \mathrm{H}, \mathrm{v}$ are functions of $\mu$ permeability.

\section{Permittivity of MHD antenna}

As per Maxwell's equation

$\nabla \times \mathrm{E}=-\mu \frac{\delta \mathrm{H}^{-1}}{\mathrm{dt}}$ $\nabla(\nabla . \mathrm{E})-\nabla^{2} \mathrm{E}=-\mu \frac{\delta}{\mathrm{dt}}(\nabla \times \vec{H})$

And

$\nabla \times \vec{H}=\mathrm{J}+\frac{\delta}{\mathrm{dt}}(\epsilon \vec{E})$

On substitution, we get

$$
\begin{gathered}
\nabla^{2} \mathrm{E}-\mu \frac{\delta}{\mathrm{dt}}\left(\mathrm{J}+\frac{\delta}{\mathrm{dt}}(\epsilon \vec{E})+\nabla \sum_{a, b} E_{a} E_{b, a} \frac{\delta \mathrm{f}}{\delta E_{b}}=0\right. \\
\frac{\delta}{\mathrm{dt}}(\epsilon \vec{E})=\frac{\delta}{\mathrm{dt}}(\epsilon \vec{E})+\epsilon \frac{\delta \vec{E}}{\mathrm{dt}}=\sum_{a} \frac{\delta \epsilon}{\delta E_{a}} E_{a}, t \vec{E}+\epsilon \frac{\delta \mathrm{E}}{\mathrm{dt}} \\
J=\sigma(\vec{E}+\vec{v} \times \vec{B}) \\
\nabla^{2} \mathrm{E}-\mu \frac{\delta^{2}}{d t^{2}}(\epsilon \vec{E})-\mu \sigma \frac{\delta}{\mathrm{dt}}(\vec{E}+\mu v \times H) \\
\nabla^{2} \mathrm{E}-\mu \frac{\delta^{2}}{d t^{2}}(\epsilon \vec{E})-\mu \sigma \frac{\delta}{\mathrm{dt}}(\vec{E}+\mu(v \times H)) \\
+\vec{\nabla} \sum_{a, b} E_{a} E_{b, a} \frac{\delta \mathrm{f}}{\delta E_{b}} \\
=0
\end{gathered}
$$

When, $\epsilon$ is a function of (E, $\mathrm{H}, v)$

$\sum_{a, b} \frac{\delta \epsilon}{\delta E_{a}} E_{a, t} E_{b}+\epsilon \frac{\delta E_{b}}{\mathrm{dt}}+\sigma(\vec{E}+\vec{v} \times \vec{B})_{b}=\sum_{a, c} e_{a b c} H_{c, a}+$ $\sum_{a}\left(\epsilon \delta_{b a}+\right.$

$\left.\mathrm{T}_{b a}\right) \mathrm{E}_{a, t}$

$$
\begin{gathered}
\text { here } \mathrm{T}_{b a}=E_{b} \frac{\delta \epsilon}{\delta E_{a}} \text { or } \\
=-\sigma(E b+v \mathrm{x} B)_{b}+(\nabla \mathrm{xH})_{b} \\
e_{a b c}=\text { Cyclic Tensor }
\end{gathered}
$$

$\epsilon\left(E_{1}, E_{2}, E_{3}\right)$ are electric field dipole moments.

Call $\log \epsilon=$ function $\mathrm{F}(\vec{E})$, hence $\epsilon(\vec{E})=e^{F(\vec{E})}$

On Summed over a, b and inverse of matrix

$\lambda_{k}$ are known values and $\varphi_{k}$ are test functions.

Permittivity can be solved by variational method or by measurement method or test function methods.

$$
\epsilon=\sum_{k=1}^{n}{ }_{k} \lambda_{k} \varphi_{k}(\vec{E})
$$

$$
\sum_{k=1}^{n} \lambda_{k} \varphi_{k}(\vec{E}) I+\sum_{k} \lambda_{k} E_{\alpha} \frac{\delta \varphi_{k}(\vec{E})}{\delta E_{\beta}}
$$

Let

$$
\left(\left(E_{\alpha} \frac{\delta \varphi_{k}(\vec{E})}{\delta E_{\beta}}\right)\right)=C_{\alpha \beta}{ }^{(K)} \text { is matrix of element } \alpha, \beta
$$

upto $\mathrm{k}$

Hence

$\left(\sum_{k} \lambda_{k} \varphi_{k} I+\sum_{k} \lambda_{k} C_{\alpha \beta}{ }^{(K)}\right)^{-1}$ 


$$
\begin{gathered}
F\left(\left\{\lambda_{k}\right\}\right)=\int_{[0, T] x V}^{\cdot}\left(\mid \frac{\delta \epsilon}{\mathrm{dt}}-\left(\begin{array}{cc}
\sum_{k=1}^{n} \lambda_{k} & \varphi_{k} I+ \\
\left.C_{\alpha \beta}{ }^{(K)}\right)^{-1}+(\vec{E}+\mu v \times \mathrm{x} H)+\nabla \times\left. H\right|^{2} \delta t d^{3} r
\end{array}\right.\right.
\end{gathered}
$$

Permittivity

solution can be worked out by difference method or test function method.

$$
\epsilon(\vec{E})=\sum_{k=1}^{n} \widehat{\lambda_{\mathbf{k}}} \varphi_{k}(\vec{E})
$$

\section{PROTOTYPE DEVELOPMENT}

Two cylindrical tubes of PPR Pipes of diameters of $10.5 \mathrm{~cm}$ and $6.2 \mathrm{~cm}$ with $6.5 \mathrm{~cm}$ lengths were mounted on copper coated plate $35 \mathrm{~cm}$ diameter circular sheet as ground plane. SMA connector was mounted on outer tube for RF input. Two electrodes tin electrodes of size $1.2 \mathrm{~cm} \mathrm{x} 4.1 \mathrm{~cm}$ mounted on the inside wall of bigger tube having direct contact with the conducting fluid. DC voltage was given with DC source, 5-25 $\mathrm{V}$ with BIAS TEE arrangement. Copper coated plate $0.2 \mathrm{~mm}$ thickness was connected $0.9 \mathrm{~cm}$ to ground of SMA connector. Copper coated circular plate was used to create ground plane. The probe of $.08 \mathrm{~cm}$ in diameter and $0.75 \mathrm{~cm}$ protruded from SMA connector was inserted in such way, that it to makes direct contact with the conducting fluid. Two permanent bar magnets of $15 \mathrm{~cm} \times 4 \mathrm{~cm} \times 2 \mathrm{~cm}$ were placed perpendicular to the electric field to produce Lorentz force to create fluid flow. Inside the tube saline water having 1200 - 9000 TDS (total dissolved salt) value was used in ionised state, to produce radiations. $300 \mathrm{ml}$ volume of saline water was used for perfect impedance matching at resonant frequency. The RF signal was given by network analyser through SMA connector with mixed DC voltage to the fluid. S11prameters were recorded as per fig 3-5.

\section{Detailed Description of MHD ANTENNA}

In this antenna, only ionised currents contribute to radiate energy in conducting fluid. Radiating resistance and resonant frequency shall depend on shape of fluid inside the tube and nano particles of the fluid. The tube was applied to external magnetic field which interacts with electric field to produce Lorentz forces, resulting in fluid flow with velocity v. Now there are three main fields i.e. electric field, magnetic field and velocity fields, which are responsible for the possible radiations. The radiated energy and its pattern are function of RF input excitation, fields applied, fluid shape and nano particle of fluid. Hence an adaptive mechanism can be built in antenna to produce versatility in radiation pattern and broad band effects, due to dynamic material perturbations.

We have formulated various equations (1-12) to focus on physics of the design analysis of an MHD antenna. Here we describe complete mechanism for beam formation, radiating patterns and resonance. Radiation pattern in the far fields depends not only on electromagnetic field but also on fluid velocity field. We have described mathematical relations of permeability as the function of $\mathrm{E}, \mathrm{H}$ and $\mathrm{v}$, when conductivity and permittivity are kept constant. With proper filtering techniques, MHD antenna can made to operate at one single frequency. Fluid shape with fields decides resonant frequency. The effective permeability can be controlled by applying a static magnetic field. This leads to the possibility of magnetically tuning of polarisation of the antenna. Polarisation tuning of antenna was measured as a function of strength for magnetisation parallel to the $\mathrm{x}$ - and $\mathrm{y}$-directions. The effects of magnetic bias on antenna have been investigated. The principle of this class of antenna is essentially that of a dielectric resonator, where salt (in solution) and electric field modifies the dielectric properties. The resonator column shape determined the operating frequency, allowing impedance match and frequency of operation to be fully tuneable. Figure 1 presents complete test set up of MHD antenna under electric and magnetic field, with RF input for S11 measurements. Figures 1-13 presents results obtained and steps of prototype development.VNA-L5230 was used to measure return loss at resonant frequency. We have varied fluid salinity, electric field, magnetic field and fluid height for all possible radiation measurement in experimentations. We have recorded return loss and radiation patterns for all possible combinations as mentioned in tables 1-2.

This antenna with conducting fluid may have multiple advantages viz reconfigurability, frequency agility, polarisation agility, broadband and beam steering capability. Here, we developed control of polarization with magnetic field biasing, frequency control with fluid height and return loss with electric field control. Non reflecting stealth property of the fluid, when no field presents, makes it most suitable for military applications.

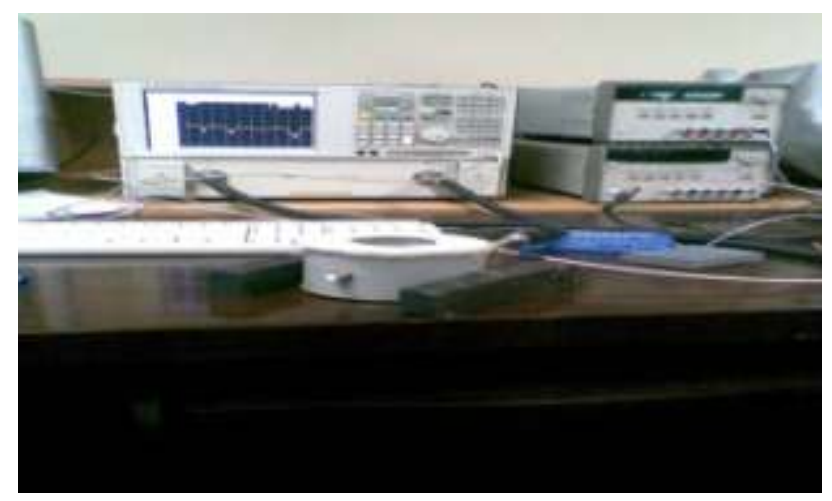

Fig 1 Measurements of return loss/resonant frequency, MHD antenna with VNA and power supply with Bias TEE

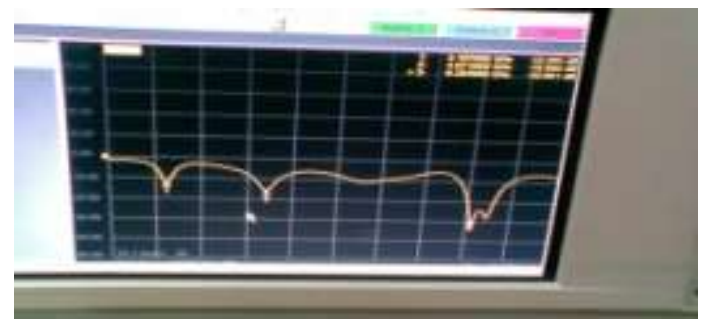

Fig 2 Return loss $-33.1 \mathrm{~dB}$ at resonant freq $8.59 \mathrm{GHz}$, when TDS 9000, electric field Applied $15 \mathrm{~V}$, DC with permanent magnetic field. 


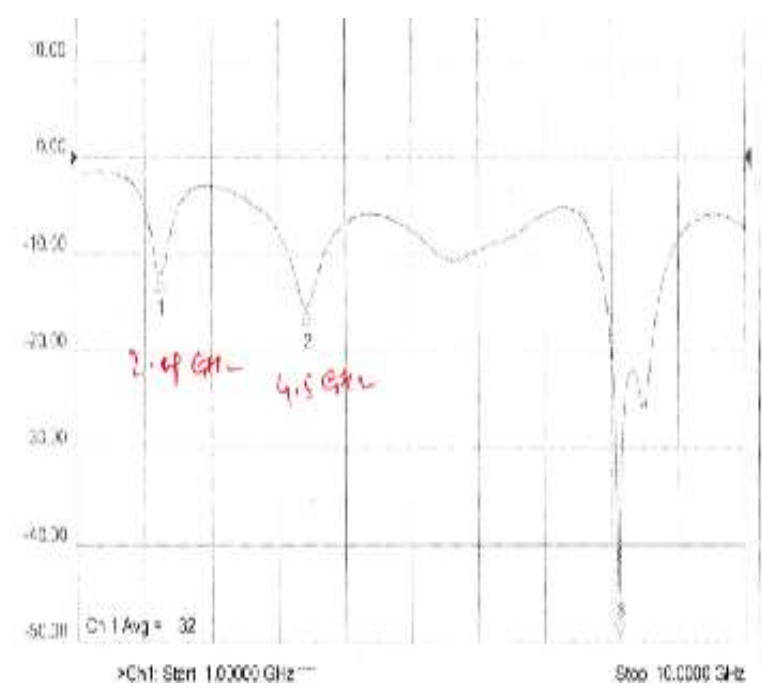

Fig 3. Return loss $-51.1 \mathrm{~dB}$ at resonant freq $8.59 \mathrm{GHz}$, when TDS 9000 , electric field applied $17 \mathrm{~V}, \mathrm{DC}$ with permanent magnetic field.

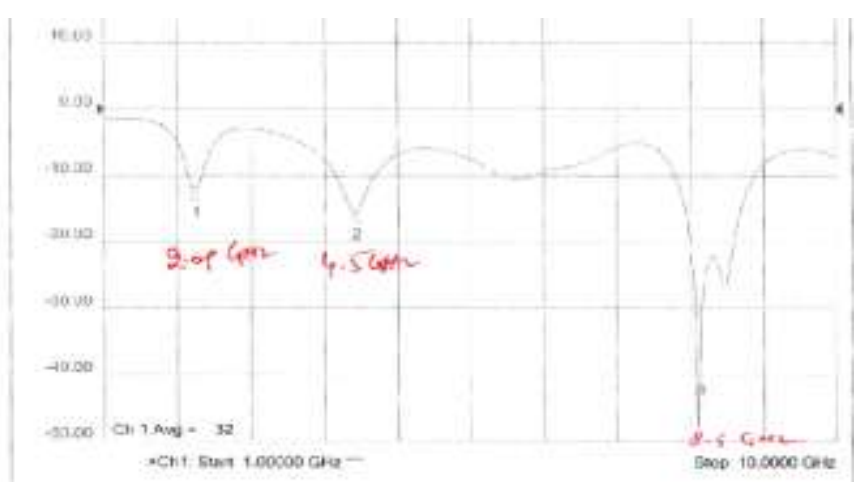

Fig 4 return loss $-49.1 \mathrm{~dB}$ at resonant freq $8.59 \mathrm{GHz}$, when TDS 9000,electric field applied 16.9 V, DC with permanent Magnetic field.

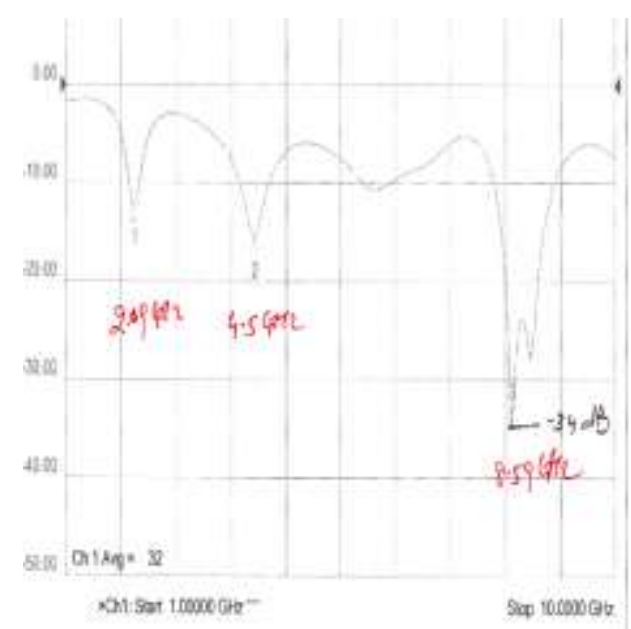

Fig 5 return loss $-34.1 \mathrm{~dB}$ at resonant freq $8.59 \mathrm{GHz}$, when TDS 9000 ,electric field applied $15.0 \mathrm{~V}$, DC with permanent magnetic field.

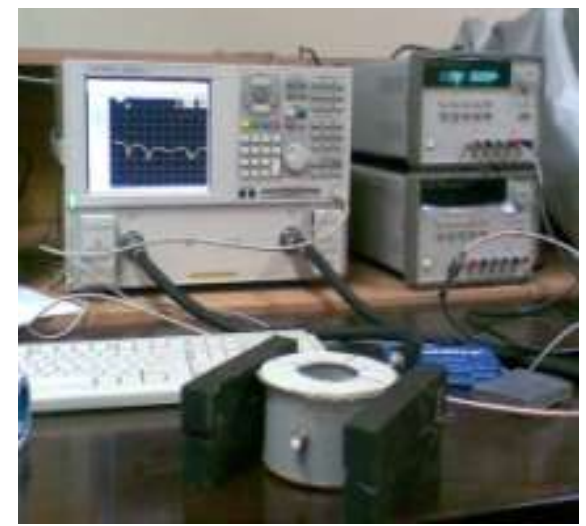

Fig 6 complete set for measurements of VSWR on MHD antenna with additional magnetic field

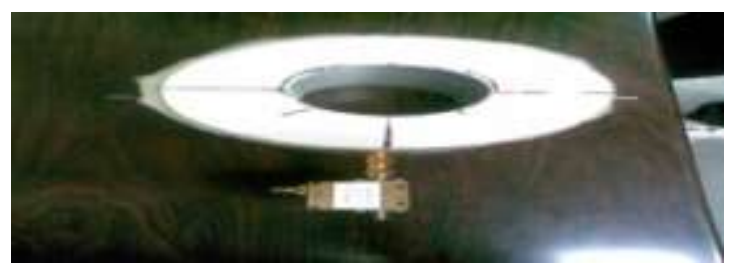

Fig 7 MHD antenna with Bias TEE

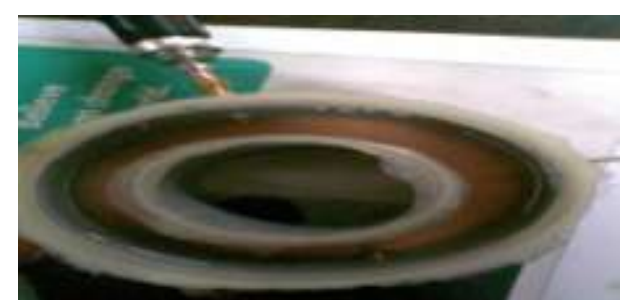

Fig 8 Fabricated MHD Antenna, SMA connector and filled Saline water top view

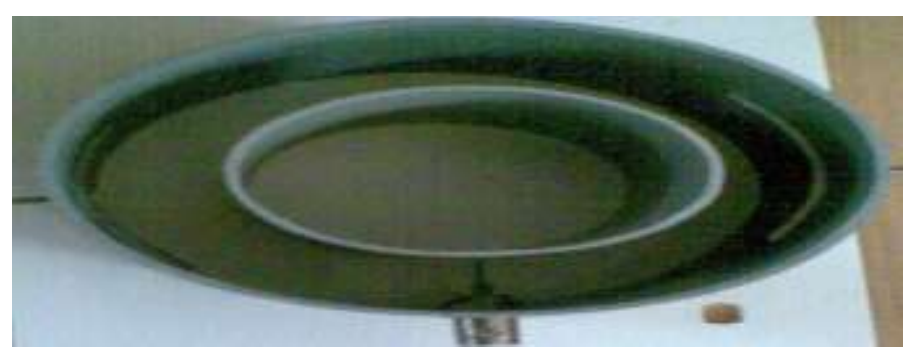

Fig 9 view of fabricated MHD antenna without ground plane

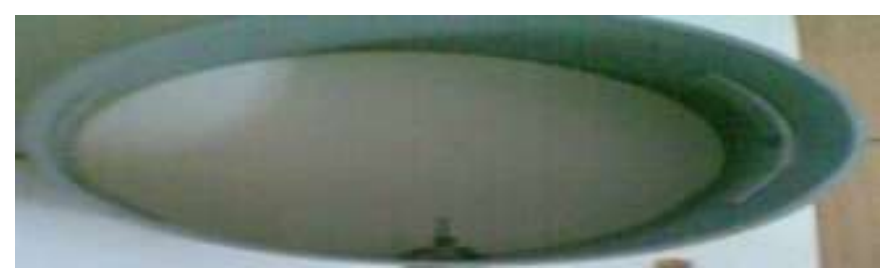

Fig 10 View of outer part of MHD antenna Tube with two tin electrodes attached 


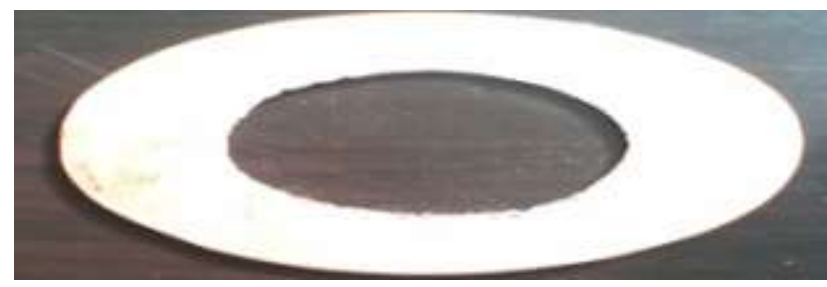

Fig 11 view of fabricated ground plane of MHD antenna with copper coating.

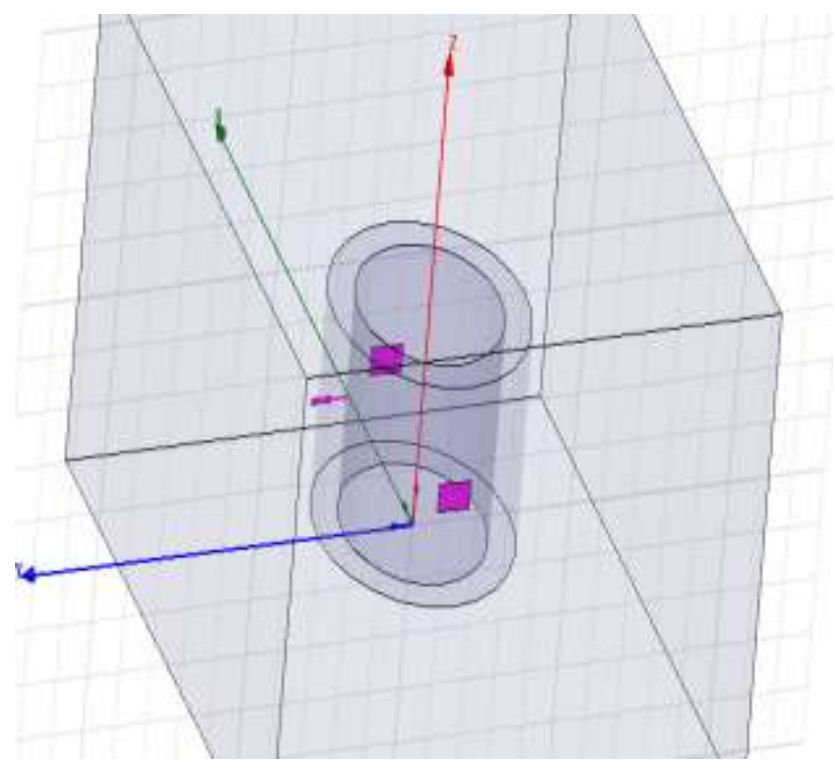

Fig 12 HFSS generated cylindrical antenna

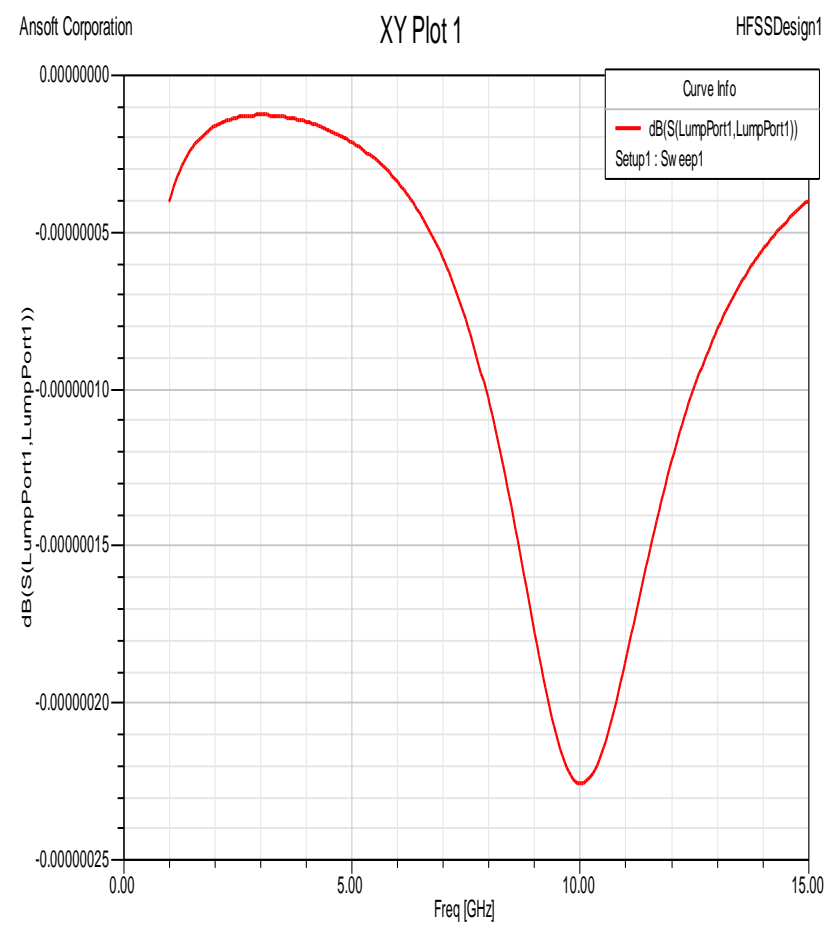

Fig 13 HFSS simulated resonant $10 \mathrm{GHz}$ frequency
Table 1

$\begin{array}{llll}\text { Freq } & \text { TDS } & \text { Electric field } & \text { Return Loss } \\ 8.59 \mathrm{GHz} & 9000 & 17.0 \mathrm{~V} & -51.1 \mathrm{~dB} \\ 4.50 \mathrm{GHz} & \text { same } & \text { same } & -16.7 \mathrm{~dB}\end{array}$

$2.09 \mathrm{GHz}$ same same $-11.7 \mathrm{~dB}$

Table 2

$8.59 \mathrm{GHz} 9000$

$4.50 \mathrm{GHz}$ same

$2.09 \mathrm{GHz}$ same

$\begin{array}{ll}16.9 \mathrm{~V} & -49.1 \mathrm{~dB} \\ \text { same } & -16.1 \mathrm{~dB} \\ \text { same } & -11.9 \mathrm{~dB}\end{array}$

Table 3

$8.59 \mathrm{GHz} 9000 \quad 15.0 \mathrm{~V} \quad-39.1 \mathrm{~dB}$

$4.50 \mathrm{GHz}$ same same $-16.2 \mathrm{~dB}$

$2.09 \mathrm{GHz}$ same same $-11.7 \mathrm{~dB}$

Table 4

$8.59 \mathrm{GHz} 9000 \quad 13.2 \mathrm{~V} \quad-35.1 \mathrm{~dB}$

$4.50 \mathrm{GHz}$ same same $\quad-15.2 \mathrm{~dB}$

$2.09 \mathrm{GHz}$ same same $-11.1 \mathrm{~dB}$

Table 5

$\begin{array}{ccc}\text { TDS } & \text { Fluid Height } & \text { Resonant frequency } \\ 5000 & 3.5 \mathrm{~cm} \quad 4.59 \mathrm{GHz} & \end{array}$

$\begin{array}{ccc}7000 & 6.0 \mathrm{~cm} \quad 8.58 \mathrm{GHz}\end{array}$

For measuring return loss, VSWR and resonant frequency, we have used PNA-L Network analyser 10-40 GHz with DC power supply. The resonant frequency for which antenna has been fabricated destined was 8.59Ghz. However we have frequency agility and reconfigurability in this antenna. Fluid column height was varied from $2.5 \mathrm{~cm}$ to $6 \mathrm{~cm}$ and electric field was varied from $2 \mathrm{~V} \mathrm{DC}$ to $17 \mathrm{~V} \mathrm{DC}$, relevent results of VSWR and Return loss were recorded.

We measured return loss by Agilent VNA(vector network analyser), the fluid tube height was kept fixed to $6 \mathrm{~cm}$ and resonant frequency to $8.58 \mathrm{Ghz}$, Dc voltage varied from $9 \mathrm{~V}$ to $17 \mathrm{~V}$. Return loss found varying proportionately to electric and magnetic field. Also when TDS was increased from 200 to 9000 significant improvement in return loss were observed. Mixed signal of DC and RF freq were fed to SMA connector of antenna through Bias TEE. This test set up extended safety to the network analyser.

\section{CONCLUSION}

It was observed from the measured results that there is significant improvement in return loss when salinity of fluid is enhanced. Also return loss improved due electric and magnetic fields intensity. We have observed that electric field have significant impact on return loss, these measured results are placed in tables1-5. Bias TEE was used to feed mixed signal from the same port .Return loss was significantly high at $17 \mathrm{~V}$, DC. Height of fluid tube (fluid shape), nano particles of fluid contribute to form resonant frequency of fluid antenna. When height of fluid was $3.5 \mathrm{~cm}$, our antenna resonated at $4.59 \mathrm{GHz}$ and when height of fluid increased to $6.0 \mathrm{~cm}$, same antenna resonated at $8.59 \mathrm{GHz}$. We have also simulated taking saline water as dielectric in HFSS antenna software for resonant frequency evaluation as per fig 12-13. We could thus achieved reconfigurability and frequency agility in this antenna. It has stealth property, as reflector is voltage dependent, hence can be 
most suitable for Military applications. We can also use this antenna as MIMO (multiple input outputs). More work towards micro-fluidic frequency reconfiguration, fluidic tuning of matching networks for bandwidth enhancement need to be explored.

As a Future work, we will investigate radiation patterns as a special case to this cylindrical antenna with detailed physics involved.

\section{ACKNOWLEDGEMENT \& BIOGRAPHY}

Prof Raj Senani, Director NSIT, who inspired me for this research work and enriched with all necessary resources required in the college. I extends special thanks to my lab technician Mr Raman, who helped me in lab for developing this prototype MHD antenna.

\section{REFERENCES}

[1] Rajveer S Yaduvanshi and Harish Parthasarathy, "Design, Development and Simulations of MHD Equations with its proto type implementations"(IJACSA) International Journal of Advanced Computer Science and Applications,Vol. 1, No. 4, October 2010.

[2] Rajveer S Yaduvanshi and Harish Parthasarathy, "EM Wave transport 2D and 3D investigations" (IJACSA) International Journal of Advanced Computer Science and Applications, Vol. 1, No. 6, December 2010.

[3] Rajveer S Yaduvanshi and Harish Parthasarathy, "Exact solution of 3D Magnetohydrodynamic system with nonlinearity analysis" IJATIT, Jan 2011.

[4] EM Lifshitz and LD Landau, "Theory of Elasticity, 3rd edition Elsevier.

[5] EM Lifshitz and LD Landau," Classical theory of fields , 4th edition Elsevier.

[6] Bahadir, A.R. and T. Abbasov (2005), "A numerical investigation of the liquid flow velocity over an infinity plate which is taking place in a magnetic field" International journal of applied electromagnetic and mechanics 21, 1-10.

[7] EM Lifshitz and LD Landau, "Electrodynamics of continuous media" Butterworth-Heinemann.

[8] EM Lifshitz and LD Landau, "Fluid Mechanics" Vol. 6 Butterworth -
Heinemann.

[9] EM Lifshitz and LD Landau, "Theory of Fields" Vol. 2 ButterworthHeinemann.

[10] JD Jackson, "Classical Electrodynamics" third volume, Wiley

[11] CA Balanis, "Antenna Theory", Wiley.

[12] Gregory H. Huff, Member, IEEE, David L. Rolando, Student Member, IEEE, Phillip Walters, Student Member, IEEE and Jacob McDonald, "A Frequency Reconfigurable Dielectric Resonator Antenna using Colloidal Dispersions" IEEE ANTENNAS AND WIRELESS PROPAGATION LETTERS,VOL. 9, 2010.

\section{AUthors PRofile}

Author: Rajveer S Yaduvanshi, Asst Professor

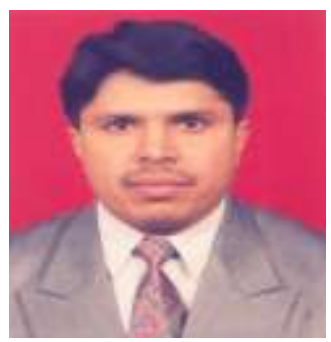

Author has 21 years of teaching and research experience. He has successfully implemented fighter aircraft arresting barrier projects at select flying stations of Indian Air Force. He has worked on Indigenization projects of 3D radars at BEL and visited France for Radar Modernisation as Senior Scientific Officer in Min of Defence. Currently he is working on MHD projects. He is teaching in ECE Deptt. of AIT, Govt of Delhi-110031. He is fellow member of IETE. His research includes Ten number of research papers published in international journals and conferences.

Co- Author: Prof Harish Parthasarathy is an eminent academician and great researcher. He is professor in ECE Deptt. at NSIT, Dwarka, Delhi. He has extra ordinary research instinct and a great book writer in the field of signal processing. He has published more than ten books and has been associated with seven PhDs scholars in ECE Deptt of NSIT, Delhi.

Co-author: Prof Asok De is an eminent researcher and effective administrator. He has set up an engineering college of repute under Delhi Government. Currently he is Principal of AIT. His research interests are micro strip antenna design. 